http://jmscr.igmpublication.org/home/ ISSN (e)-2347-176x ISSN (p) 2455-0450

crossref DOI: https://dx.doi.org/10.18535/jmscr/v8i7.81

Journal Of Medical Science And Clinical Research

\title{
To study the effect of Olmesartan medoxamil in patients with hypertension
}

\author{
Author \\ Dr Meghraj Singh Patel (MBBS, MD, DM) \\ Consultant Neurologist, Siddhanta Red Cross Super Speciality Hospital, Bhopal (M.P.), India \\ Corresponding Author \\ Dr Meghraj Singh Patel (MBBS, MD, DM)
}

\begin{abstract}
Introduction: Hypertension (high blood pressure) affects approximately 1 billion individuals worldwide. Epidemiological studies show a steadily increasing trend in hypertension prevalence over the last 40 years, more in urban than in the rural areas. Newer antihypertensive agents are constantly being introduced and heavily promoted. At present several Angiotensin receptor blockers (ARBs) are in use for the treatment of hypertension, heart failure and diabetic nephropathy. The newest agent in the class is Olmesartan medoxomil approved by FDA in April 2002 and widely used since then. By this study we will analyze the clinical outcome of Olmesartan medoxamil in patient with hypertension.

Objectives: To study the changes in BP, ECG and ECHO finding from baseline after giving Olmesartan. Material \& Methods: The present prospective study was carried out on 39 cases with 18-70 years age group diagnosed as hypertension (stage I and stage II - JNC7). After taking written informed consent detail history, demographic and clinical data, past medical and family history; presenting symptoms and signs; physical examination findings, BP, Hematological and Biochemical parameters ECG and ECHO findings were recorded in preformed proformas. Olmesartan $20 \mathrm{mg} /$ day was given as starting dose and further increased if BP not controlled. All cases will be registered and followed up after at 3 month and 6-month. Blood pressure, ECG and, left ventricle (LV) dimensions and Left ventricular mass index (LVMI) by Echocardiography will be measured and data will be compared by appropriate statistical tests.

Result: In our study mean reduction in SBP and DBP from baseline to after three months of treatment was $6.72 \pm 7.54(P<0.0001)$ and $6.94 \pm 6.16(P<0.0001)$ and after 6 months was $10.16 \pm 9.57(P<0.001)$ and $9.38 \pm 6.56(P<0.001)$. On comparison between mean LVMI at baseline (43.23\%) and after 3 months $(42.65 \%)$ mean reduction was $0.57 \pm 1.01(P=0.12$, not significant) after 6 month $(41.30 \%)$ the mean reduction was $1.93 \pm 2.10(P=0.025$, significant $)$.

Conclusion: Olmesartan medoxomil at the recommended dose of $20 \mathrm{mg}$ once daily will be effective in treatment of essential hypertension but was not effective in regression of ECG based left ventricular hypertrophy. Olmesartan medoxomil after treatment reduced the left ventricular mass index which was statistically significant. This study will be served as a pilot study for future research.

Keywords: Olmesartan, Hypertension, Left ventricular hypertrophy.
\end{abstract}

\section{Introduction}

Hypertension (high blood pressure) affects approximately 1 billion individuals worldwide.
Epidemiological studies show a steadily increasing trend in hypertension prevalence over the last 40 years, more in urban than in the rural 
areas. Hypertension is directly responsible for $57 \%$ of all stroke deaths and $24 \%$ of all coronary heart disease deaths in India. ${ }^{1}$ Current treatment guidelines recommend that $\mathrm{BP}$ should be reduced to $<130 / 80 \mathrm{~mm} \mathrm{Hg}$ in patients with diabetes mellitus or chronic renal disease and to $<140 / 90 \mathrm{~mm} \mathrm{Hg}$ in all others. ${ }^{2}$ However, many patients with essential hypertension fail to achieve these targets with currently recommended treatments. Because the treatment of hypertension is among the leading indications for the use of drugs, new agents constantly are being introduced and heavily promoted. The choice of drugs is one of the factors that affects the efficacy of therapy.

At present several Angiotensin receptor blockers (ARBs) are in use for the treatment of hypertension, heart failure and diabetic nephropathy. The newest agent in the class is Olmesartan medoxomil approved by FDA in April 2002 and widely used since then. Thus, by this study we will analyse the clinical outcome of Olmesartan medoxamil in patient with hypertension.

\section{Aims \& Objectives}

1. To study the change in BP from baseline after giving Olmesartan

2. To study the change in ECG findings from baseline after giving Olmesartan.

3. To study the change in ECHO findings from baseline after giving Olmesartan.

\section{Materials and Methods}

The present prospective study was carried out on 39 cases with 18-70 years age group diagnosed as hypertension (stage I and stage II - JNC7) ${ }^{2}$ in the district Gwalior (M.P.) and surrounding areas between the year2007-2008. Following cases were excluded in our study:

- Severe hypertension(DBP $>110 \mathrm{~mm} \mathrm{Hg}$ or SBP > 200mmHg)

- History of secondary hypertension including renal disease, pheochromocytoma, Cushing's disease.

- Pregnant or lactating female.
- History of myocardial infarction (MI), Percutaneous transluminal coronary angiography (PTCA), Coronary artery bypass grafting (CABG), unstable angina pectoris or an episode of heart failure requiring hospitalization.

- Previous history of CVA or TIA.

- History of allergy to Olmesartan medoxomil.

- Any serious disorder, including pulmonary, hepaticrenal, gastrointestinal, uncontrolled endocrine/metabolic hematological/ oncological, neurological, and psychiatric disease that would interfere with the conduct of the study or interpretation of data.

- Diabetes mellitus patients.

After taking written informed consent detail history, demographic and clinical data, including past medical and family history; presenting symptoms and signs; physical examination findings, BP, anthropometric measurement, body mass index, and systemic examination findings were recorded in preformed proformas.

Hematological and Biochemical assessment included hemoglobin, total leukocyte count, ESR, fasting and postprandial blood sugar, blood urea, serum creatinine, serum bilirubin and lipid profile were done and finding noted.

BP will be taken in the upper arm in the patients comfortably seated with the back and arm supported, the leg uncrossed and the upper arm at the level of heart. Presence of hypertension will be defined as per the Joint National Committee (JNC) VII criteria.

Standard 12 lead ECGs will be recorded at 25 $\mathrm{mm} / \mathrm{s}$ and $0.1 \mathrm{mv} / \mathrm{mm}$ standardization with equipment whose frequency response characteristics met recommendation of American Heart Association. In the ECG Sokolow- Lyon index is calculated (sum of the amplitude of the $S$ wave in in lead V1 and the R wave in lead V5 or V6). If it is equal to or more then $3.5 \mathrm{mV}$ then it is considered as positive. If it less than $3.5 \mathrm{mv}$ then it is considered as negative. 
2D guided M-mode echocardiogram of Left ventricle at the tip of mitral leaflet using the parasternal view done to measure left ventricular dimension including interventricular septal thickness in diastole (IVSTD), posterior wall thickness in diastole (PWTD) and left ventricular internal diameter at diastole (LVIDD), will be measured from 2D guided M-mode echocardiograms of Left ventricle at the tip of mitral leaflet using the parasternal view.

Olmesartan will be given to the patients with starting dose of $20 \mathrm{mg} /$ day, if blood pressure not controlled in follow up period the dose may be increased. All patients will be registered and followed up after a period of 3 month and 6 months from the day of registration. At 3 month and 6-month blood pressure, ECG and, left ventricle (LV) dimensions and Left ventricular mass index (LVMI) by Echocardiography will be measured and data will be compared by appropriate statistical tests.

\section{Statistician Analysis}

Values are expressed as the Mean+/- standard deviation paired ' $t$ ' test was used to compare values from the baseline run-in period with values obtained during the treatment period. The level of significance was set at $\mathrm{p}<0.05$ for all comparisons.

\section{Results}

As shown in table 1, in our study $64.10 \%$ cases were male while female are only $35.895 \%$ making male: Female ratio is 1.78 which is in favor of male. Maximum number of cases were in the age group $61-75$ years $(46.15 \%)$ followed by age group 46-60 years (41.02\%).Main presenting complaint was headache $(73.92 \%)$ followed by weakness (25.64\%) and ghabrahat (23.07\%). Duration of hypertension was less than 5 years in $56.41 \%$ of cases. Family history of hypertension present in $66.67 \%$ cases. History of smoking and tobacco chewing were most common findings ( $20.51 \%$ each) followed by physical inactivity and high salt intake (12.82\% each).The $51.28 \%$ patients belong to the normal BMI group (18.524.9) followed by that of overweight category (38.46\%). Amongst male cases $68 \%$ were of stage I whereas $32 \%$ were of stage II amongst female $64.28 \%$ were of stage I and $35.71 \%$ were of stage II hypertension.

As described in Table $2 \& 3$, ECG findings of LVH present in $7.69 \%$ of cases with stage II hypertension at baseline which was decreased to $5.55 \%$ after three month and $2.81 \%$ after 6 months of giving Olmesartan.

As in table 4, baseline significant LVMI was present in $82.05 \%$ cases, they all were of stage II hypertension.

As described in table 5, on comparison between SBP and DBP at baseline and after three months mean reduction was $6.72 \pm 7.54$ with $\mathrm{P}<0.0001$ and $6.94 \pm 6.16$ with $\mathrm{P}<0.0001$, after 6 month the mean reduction in SBP was $10.16 \pm 9.57$ with $\mathrm{P}<$ 0.001 and $9.38 \pm 6.56$ with $\mathrm{P}<0.001$.On comparison between mean LVMI at baseline and after 3 months mean reduction was $0.57 \pm 1.01$ with $\mathrm{P}=0.12$ (not significant) after 6 month the mean reduction was $1.93 \pm 2.10$ with $\mathrm{P}=0.025$ (significant).

Table 1: Distribution of cases according to demographic and clinical profile:

\begin{tabular}{|l|c|c|c|c|}
\hline \multicolumn{2}{|c|}{ Variables } & Male & Female & Total \\
\hline Sex & & $25(65.41 \%)$ & $14(35.89 \%)$ & 0 \\
\hline \multirow{3}{*}{ Age Group } & $18-30$ & $0(0 \%)$ & $0(0 \%)$ & $0(0 \%)$ \\
\cline { 2 - 5 } & $31-45$ & $3(12 \%)$ & $2(14.28 \%)$ & $5(12.82 \%)$ \\
\cline { 2 - 5 } & $46-60$ & $12(48 \%)$ & $4(28.57 \%)$ & $16(41.02 \%)$ \\
\cline { 2 - 5 } & $61-75$ & $10(40 \%)$ & $8(57.14 \%)$ & $18(46.15 \%)$ \\
\hline Presenting Compaints & Headache & $18(72 \%)$ & $12(85.71 \%)$ & $30(76.92 \%)$ \\
\cline { 2 - 5 } & Ghabarahat & $5(20 \%)$ & $4(28.71 \%)$ & $9(23.07 \%)$ \\
\cline { 2 - 5 } & Weakness & $5(20 \%)$ & $5(35.71 \%)$ & $10(25.64 \%)$ \\
\cline { 2 - 5 } & Easy Fatigue & $3(12 \%)$ & $3(21.42 \%)$ & $6(15.38 \%)$ \\
\cline { 2 - 5 } & Chest Pain & $3(12 \%)$ & $1(7.14 \%)$ & $4(10.25 \%)$ \\
\hline
\end{tabular}




\begin{tabular}{|l|c|c|c|c|}
\hline & Others & $2(8 \%)$ & $1(7.14 \%)$ & $3(7.69 \%)$ \\
\hline Past History (Duration) & $\leq 5$ & $14(56 \%)$ & $8(57.14 \%)$ & $22(56.41 \%)$ \\
\cline { 2 - 5 } & $\geq 5$ & $11(44 \%)$ & $6(42.85 \%)$ & $17(43.58 \%)$ \\
\hline Family History of & & $18(72 \%)$ & $8(57.14 \%)$ & $26(66.67 \%)$ \\
\hline Hypertension & Present & $5(20 \%)$ & $6(42.85 \%)$ & $11(28.20 \%)$ \\
\cline { 2 - 5 } & Absent & $8(32 \%)$ & 0 & $8(20.51 \%)$ \\
\cline { 2 - 5 } & SMOKING & $4(16 \%)$ & 0 & $4(10.25 \%)$ \\
\cline { 2 - 5 } & Alcoholic Intake & $6(24 \%)$ & $2(14.28 \%)$ & $8(20.51 \%)$ \\
\cline { 2 - 5 } & Tabacco Chewing & $4(16 \%)$ & $1(7.14 \%)$ & $5(12.82 \%)$ \\
\cline { 2 - 5 } & Physical Inactivity & $2(8 \%)$ & $1(7.14 \%)$ & $3(7.69 \%)$ \\
\cline { 2 - 5 } & Physosocial Stress & $3(12 \%)$ & $2(14.28 \%)$ & $5(12.82 \%)$ \\
\cline { 2 - 5 } & High Salt Intake & $10(40 \%)$ & $8(57.14 \%)$ & $18(46.15 \%)$ \\
\cline { 2 - 5 } & Not Significant & $15(60 \%)$ & $5(35.71 \%)$ & $20(51.28 \%)$ \\
\hline \multirow{3}{*}{ BMI } & $18.5-24.9$ & $8(32 \%)$ & $7(50 \%)$ & $15(38.46 \%)$ \\
\cline { 2 - 5 } & $25-29.9$ & $2(8 \%)$ & $2(14.28 \%)$ & $4(10.25 \%)$ \\
\cline { 2 - 5 } & $30-39.9$ & $17(68 \%)$ & $9(64.28 \%)$ & $26(66.66 \%)$ \\
\hline stage of hypertension & Stage I & $8(32 \%)$ & $5(35.71 \%)$ & $13(33.33 \%)$ \\
\cline { 2 - 5 } & Stage II & & \\
\cline { 2 - 5 } & & & & \\
\hline
\end{tabular}

Table 2: Distribution of cases according to staging of hypertension and Electrocardiography finding of Left Ventricular Hypertrophy

\begin{tabular}{|c|c|c|c|c|c|c|c|c|c|c|}
\hline \multirow{3}{*}{$\begin{array}{l}\text { LVH by } \\
\text { ECG }\end{array}$} & \multicolumn{4}{|c|}{ Stage I $(n=26)$} & \multicolumn{4}{|c|}{ Stage II $(n=13)$} & \multicolumn{2}{|c|}{ Total $(n=39)$} \\
\hline & \multicolumn{2}{|c|}{ Male $(\mathrm{n}=17)$} & \multicolumn{2}{|c|}{ Female $(\mathrm{n}=9)$} & \multicolumn{2}{|c|}{$\operatorname{Male}(\mathrm{n}=8)$} & \multicolumn{2}{|c|}{ Female $(n=5)$} & \multirow[t]{2}{*}{ No. } & \multirow{2}{*}{$\%$} \\
\hline & No & $\%$ & No & $\%$ & No & $\%$ & No & $\%$ & & \\
\hline Present & 0 & 0 & 0 & 0 & 2 & 25 & 1 & 20 & 3 & 7.69 \\
\hline Absent & 17 & $100 \%$ & 9 & $100 \%$ & 6 & $75 \%$ & 4 & $80 \%$ & 36 & $92.3 \%$ \\
\hline
\end{tabular}

Table 3: Comparison of the number of patients with ECG diagnosed LVH at baseline, 3 month and 6 months after giving Olmesartan

\begin{tabular}{|l|c|c|c|c|c|c|}
\hline \multirow{2}{*}{ LVH by ECG } & \multicolumn{2}{|c|}{ Baseline (n=39) } & \multicolumn{2}{c|}{ 3 Month $(\mathbf{n}=\mathbf{3 6})$} & \multicolumn{2}{c|}{ 6 Month $(\mathbf{n}=\mathbf{3 6})$} \\
\cline { 2 - 7 } & No & $\%$ & No & $\%$ & NO & $\%$ \\
\hline Present & 3 & 7.69 & 2 & 5.55 & 1 & 2.81 \\
\hline Absent & 36 & 92.3 & 34 & 94.44 & 35 & 97.22 \\
\hline
\end{tabular}

Table 4: Showing comparison of the number of patients with normal or increased left ventricular mass Index, 3 month and 6 months follow up after giving Olmesartan

\begin{tabular}{|l|c|c|c|c|c|c|}
\hline \multirow{2}{*}{ LVMI } & \multicolumn{2}{|c|}{ Baseline $(\mathbf{n}=\mathbf{3 9})$} & \multicolumn{2}{c|}{ 3 Month $(\mathbf{n}=\mathbf{3 6})$} & \multicolumn{2}{c|}{ 6 Month $(\mathbf{n}=\mathbf{3 6})$} \\
\cline { 2 - 7 } & No & $\%$ & No & $\%$ & NO & $\%$ \\
\hline Present & 32 & 82.05 & 28 & 77.77 & 20 & 55.55 \\
\hline Absent & 7 & 17.94 & 12 & 23.22 & 16 & 45.45 \\
\hline
\end{tabular}

Table 5: Distribution of cases as per mean systolic blood pressure (SBP), diastolic blood pressure (DBP) echocardiographic left ventricular mass index (LVMI) and statistical analysis of patient during baseline, 3 month and 6 months follow up after giving Olmesartan

\begin{tabular}{|l|c|c|c|c|}
\hline Time & Number of & \multicolumn{3}{|c|}{ Mean \pm Standard Decviation } \\
\cline { 3 - 5 } Interval & patients & SBP mmHg & DBP mmhg & LVMI (Mean \pm SD) \\
\hline Baseline & 39 & $148.66 \pm 9.51$ & $91.66 \pm 7.53$ & $43.23 \pm 25.13$ \\
\hline 3 months & 36 & $141.94 \pm 5.52$ & $84.77 \pm 6.94$ & $42.65 \pm 24.73$ \\
\hline 6 months & 36 & $138.50 \pm 4.31$ & $82 \pm 5.39$ & $41.30 \pm 23.61$ \\
\hline
\end{tabular}

\section{Discussion}

The present study was carried out with aim to study the effects of Angiotensin receptor blocker Olmesartan medoxomil in patients of essential hypertension stage I and stage II as per the JNC VII criteria. As there are several drugs available for the treatment of hypertension the real challenge while choosing antihypertensive lies in 
identifying drug therapy that offer desired blood pressure control, with minimum adverse effect and is well tolerated to the patient, so that patients are willing adhere to drug therapy regimen for the long term while maintaining quality of life.

In the present study $64.10 \%$ of cases were male with female are only $35.89 \%$ the male to female ratio is 1.78 which is in favor of male may be due to increasing smoking and addiction prevalent. In female may be due to hormonal protection up to age of menopause and delay in seeking medical advice we found low rate compare to male. ${ }^{3}$ In our study number of cases increased with the increase in age maximum number of patients $(48.15 \%)$ were in age group 61-75 years. These findings of current study were comparable to the data from the 7 th report of joint national committee on hypertension suggested the prevalence of hypertension increases with advancing age to point where more half of people 60-69 years of age and approximately $3-4$ th of those 70 years of age and older are affected. ${ }^{2}$

In present study main presenting complaint was headache $(76.92 \%)$, which was similar to various sectional survey. In the study by Steward et al, headache was present in $71 \%$ of hypertensive patients who were aware of their diagnosis. ${ }^{4}$ Family history of hypertension was present in $66.67 \%$ which was consistent with the previous study by Braunwald et.al, in which genetic sharing, genetic contributions have been estimated in the range from $30-60 \% .^{5}$ In the study of Dominiczac et al the estimate of heritability based on office BPs were in the range of $20 \%$ to $40 \%$. $^{4}$

In the present study smoking was found in majority of cases including $20.51 \%$, tobacco chewing found in $20-51 \%$. History of high salt intake and physical inactivity each present in $12.82 \%$ of cases. History of alcohol intake was less common as well as history of psychosocial accounting for $10.25 \%$ and $7.69 \%$ respectively which is supported by different studies including study of Szczech et al, Beard et al and Brydon and Steptoe. ${ }^{4}$
In the present study $32 \%$ of male and $50 \%$ of female were in the overweight category which was near comparable to the Framingham study in which the incidence of hypertension was increased $46 \%$ in men and $75 \%$ in women who were overweight, defines as BMI of 25.0 to 29.9 as compared to normal weight persons. ${ }^{5}$

In the present study $66.66 \%$ of cases were of stage I and 33.335 of stage II hypertension. Increase number of cases in stage I hypertension may be seeking earlier attention to their medical problems.

In the present study ECG findings of LVH present in $7.69 \%$ cases which was comparable to the previous studies of Schmieder \& Messerli et al, which showed that LVH is identified by ECG in only $5 \%$ to $10 \%$ of hypertensive cases. ${ }^{6}$ Whereas study by Shine YJ et al ECG for diagnosis of LVH showed sensitivity of $20.0 \% .^{7}$ In the comparison of this study there were a smaller number of patients in our study with ECG diagnosed LVH. This may be as most of the patients were of stage I hypertension and already on treatment.

In our study at baseline increase in the LVMI was found in the $82.05 \%$ of the cases. These data were not comparable to the previous study of Schmieder and Messerli et al, where they found LVH by echocardiography in nearly $30 \%$ of unselected hypertension adults. ${ }^{6}$ This may due to the effect from the level of blood pressure several other factors like obesity, high dietary sodium intake, alcohol abuse, diabetes, hypercholesterolemia, genotype, polymorphism of the Angiotensin type 2 receptor gene and serum aldosterone levels were responsible for the same. Low sample size and selection of cases from tertiary care Centre was the other reason for difference in opinion.

In our study mean systolic BP at baseline was $148.66+/-\mathrm{mmHg}$, at 3 months after treatment was $141.94 \pm 5.52 \mathrm{~mm} \mathrm{Hg}$. The mean reduction was $6.72 \pm 7.54 \mathrm{mmHg}$ with $\mathrm{p}$ value $<0.0001$. Mean $\mathrm{SBP}$ at 6 months was $1138.50 \pm 4.31 \mathrm{mmHg}$ with mean reduction from baseline after treatment was 
$10.16 \pm 9.57 \mathrm{mmHg}$ with $\mathrm{p}$ value 0.0001 . Similarly, the mean DBP at baseline, 3 month and 6 month was $91.66 \pm 7.53 \mathrm{mmHg}, 84.77 \pm 6.94$ $\mathrm{mmHg}$ and $82 \pm 5.36 \mathrm{mmHg}$ respectively with mean reduction at 3 month was $6.94 \pm 6 \mathrm{mmHg}$ and at 6 month was $9.38 \pm 6.56 \mathrm{mmHg} \mathrm{p}$ value < 0.0001 for pair. The reduction in the mean SBP and mean DBP in current study was comparable to results published by Manoria et al. The result of OLMIBEST study showed the mean reduction from baseline in diastolic BP at week 8 was 11.8 $\mathrm{mmHg}$ and that in SBP $17.8 \mathrm{mmHg}$. The study even demonstrated that monotherapy with Olmesartanat the recommended daily dose (20mg) is effective in patients with mild to moderate hypertension. ${ }^{9}$ The result of current study were also comparable to the study by Neutel JM et al which was a multi-centre, randomised, placebocontrolled, double-blind clinical trials involving a total of 2,693 subjects from three centers in the US and four center in Europe were reported in a meta-analysis in 2001. The length of treatment in these studies spanned from six to 12 weeks. At the recommended starting those of $20 \mathrm{mg} / \mathrm{day}$, the mean change in DBP and SBP were-12.2 and 15.1 mmHg respectively. ${ }^{10}$ Thus, current open level treatment with Olmesartan medoxomil 20mg per day for 6 months confirmed the high antihypertensive efficacy of this regimen in the treatment of essential hypertension.

On comparison between mean LVMI at baseline and after 3 months mean reduction was $0.57 \pm 1.01$ with $p$ value of 0.12 (not significant) after 6 month the mean reduction was $1.93 \pm 2.10$ with $p$ value of 0.025 (significant).Previous study by Domenico Galzerano et al on Telmisatan for 44 weeks showed that in Telmisartan-treated patients, LV mass index was reduced by $21.9 \pm 5.9 \mathrm{~g} / \mathrm{m} 2$, which represents a $15.7 \%$ reduction. In carvedilol-treated patients, LV mass index was reduced by $12.8 \pm 3.5$ $\mathrm{g} / \mathrm{m} 2$, representing a $9.0 \%$ regression and proved that Telmisartan was significantly superior to carvedilol, $\mathrm{P}<0001$. In our study the mean reduction of LVMI was superior to the study by Domenico Galzerano. This may be because of differences in method of indexing of left ventricular mass in both the studies. ${ }^{9}$

Overall, 4 patients out 39 experienced adverse events including dizziness, headache and upper respiratory tract infections. Most adverse events were mild and were considered unrelated/unlikely related to the medication. Out 39 patients 3 patients were lost in the first follow up.

\section{Conclusion}

Olmesartan medoxomil at the recommended dose of $20 \mathrm{mg}$ once daily will be effective in treatment of essential hypertension. These results support the use of this dose regimen in the initial management of hypertensive patients. Olmesartan medoxomil was not effective in regression of ECG based left ventricular hypertrophy in patient of essential hypertension. Olmesartan medoxomil reduce the left ventricular mass index. The reduction in the LVMI was statistically significant.

\section{Funding: No Funding Sources}

Conflict of Interest: None Declared

Ethical Approval: The study was approved by the Institutional Ethics Committee

\section{References}

1. R Gupta. Trends in hypertension epidemiology in India. J Hum Hypertens 2004; Feb;18(2):73-8 doi: 10.1038/sj.jhh.1001633.

2. Aram V Chobanian, George L Bakris, Henry R Black, William C Cushman, Lee A Green, Joseph L Izzo Jr et al. The Seventh Report of the Joint National Committee on Prevention, Detection, Evaluation, and Treatment of High Blood Pressure: the JNC 7 report. JAMA 2003 May 21;289(19):2560-72 doi: 10.1001/jama.289.19.2560.

3. Anthony S. Fauci, Eugene Braunwald, Dennis L. Kasper, Stephen L. Hauser, Dan L. Longo, J. Larry Jameson Harrison's 
Principles of Internal Medicine, 17th Edition 2008.

4. Norman M Kaplan Kaplan's Clinical Hypertension, 9th edn (2005). Can J Cardiol. 2007 May 15; 23(7): 605.

5. Robert O. Bonow, Douglas L. Mann, Douglas P. Zipes, Peter Libby Braunwald's Heart Disease A Textbook of Cardiovascular Medicine 8th edition 2008.

6. Schmieder, R., Messerli, F. Hypertension and the heart. J Hum Hypertens 14, 597604 (2000). https://doi.org/10.1038/sj.jhh.1001044

7. Shin YJ, Choi E J, Yu D S et al. Electrocardiogram as a Diagnostic Method for Left Ventricular Hypertrophy. Korean J Fam Med. 2005;26(9):551-560.

8. Manoria P. Olmesartan medoxomil: a clinical review. Indian Heart Journal, 30 Apr 2006, 58(3):282-286 PMID: 19033634

9. Domenico Galzerano, Paolo Tammaro, Luca del Viscovo, Diana Lama et al Three-dimensional echocardiographic and magnetic resonance assessment of the effect of telmisartan compared with carvedilol on left ventricular mass a multicenter, randomized, longitudinal study Am J Hypertens 2005 Dec;18(12 Pt 1):1563-9. doi: 10.1016/j.amjhyper.2005.06.011.

10.Joel M, Neutel MD Clinical studies of CS866, the newest angiotensin II receptor antagonist The American Journal of Cardiology Volume 87, Issue 8, Supplement 1, 19 April 2001, Pages 37-43. 